\title{
Inferring porosity from frequency dependent attenuation in cortical bone mimicking porous media
}

\author{
$1^{\text {st }}$ R.D. White \\ Mathematics Department, CRSC \\ North Carolina State University \\ Raleigh, NC 27695-8212, USA \\ rdwhite@ncsu.edu
}

$4^{\text {th }}$ Marie Muller

Mechanical and Aerospace Engineering Department

North Carolina State University

Raleigh, NC 27695-8212, USA

mmuller2@ncsu.edu $2^{\text {nd }}$ Omid Yousefian

Mechanical and Aerospace Engineering Department

North Carolina State University

Raleigh, NC 27695-8212, USA

oyousef@ncsu.edu

\author{
$3^{\text {rd }}$ H.T. Banks \\ Mathematics Department, CRSC \\ North Carolina State University \\ Raleigh, NC 27695-8212, USA \\ htbanks@ncsu.edu
}

\begin{abstract}
Osteoporosis affects porosity in cortical bone. Quantifying levels of osteoporosis by inferring the micro-architectural properties from ultrasonic wave attenuation in cortical bone has yet to be done. In this work we use a phenomenological, power law model to describe the frequency dependent attenuation in non-absorbing porous media mimicking a simplified cortical bone structure. We optimize this model to fit data generated using a finite-difference, time domain (FDTD) numerical simulation. Model parameters are estimated using an ordinary least squares (OLS) formulation of the inverse problem. With these we determine linear, functional relationships between the model parameter estimates and the micro-architectural parameters, pore density and pore diameter. These relationships allow us to infer ranges of porosity from simulated attenuation data. Repeating this process for attenuation data collected from cortical bone samples could allow one to characterize the micro-architectural properties of bone.

Index Terms - cortical bone, porosity, attenuation, inverse
\end{abstract} problem

\section{INTRODUCTION}

Osteoporosis is a prevailing bone disease, occurs due to aging, menopause, certain medications etc. and is characterized by deterioration of bone tissue, consequently increasing bone susceptibility to fracture. The involvement of cortical bone in many osteoporotic fracture, its link to bone strength and load-bearing capacities of cortical bone make it an important target of further research [1-6]. Currently the most common method for the evaluation of osteoporosis is bone mineral density (BMD) measurement using dual X-ray absorptiometry (DXA) [7]. The correlation between increased fracture risk and the loss in bone mass makes BMD a useful diagnostic factor

Air Force Office of Scientific Research under Grant No. AFOSR FA955018-1-0457, the US Department of Education Graduate Assistance in Areas of National Need (GAANN) under Grant No. P200A120047, the National Institute of Health under Grant No. R03EB022743, the National Science Foundation Graduate Research Fellowship under Grant No. DGE-1746939.
[8]; however, due to the large overlap between bone density of individuals who develop a future fracture and the ones who do not [9], BMD is considered sub-optimal for screening. It is common for women with high fracture risk to be underdiagnosed due to fairly high BMD [10].

Osteoporosis affects the micro-structure of cortical bone and is associated with changes in the microstructure of cortical porosity including pore diameter and pore density [11], [12]. The mechanical competence of cortical bone strongly depends on these two parameters [13]. Knowledge of cortical bone microarchitecture is crucial for understanding the osteoporosis pathophysiology and to improve its treatment and diagnosis [14].

Quantitative ultrasound (QUS) is a non-invasive modality that has been used increasingly in osteoporosis assessment [15]. Many studies have been conducted to address the microarchitectural properties of bone using ultrasonic parameters in cortical bone [16-21]. Among all the parameters, ultrasonic attenuation, and its frequency dependence have been investigated the least in cortical bone. Yousefian et al. [22] proposed a phenomenological model to estimate the frequency-dependent attenuation as a function of pore diameter and pore density in structures mimicking simplified cortical bone.

This study focuses on using the phenomenological model to solve an inverse problem and estimate the pore density and diameter from the frequency-dependent ultrasonic attenuation in numerical structures of simplified cortical bone. We begin by explaining the methodology for the data collection process. Next, we introduce the mathematical and statistical models, followed by the formulation of the inverse problem using these models. We conclude with the results and related conclusions. 


\section{Methodology for Data Collection}

A finite-difference, time domain (FDTD) research freeware, SimSonic (www.simsonic.fr) [18] is used to simulate elastic waves propagating in porous media resembling cortical bone [23-25]. The media contains a distribution of fluid-filled pores (with material properties of water) within solid slabs (with material properties of pure bone) [26]. The ability of defining independently tuneable material properties at all points in space enables an understanding of the exclusive effect of the different parameters of the porosity, including pore size and density (number of pores per unit area). The range of pore diameter $(\phi)$ and density $(\rho)$ are respectively chosen as $\rho \in[3,16]$ pore $/ \mathrm{mm}^{2}$ and $\phi \in[20,100] \mu m$ according to values found in the literature [27-29]. Using a Monte Carlo method, the porous structures mimicking simplified cortical bone is generated for a given pore diameter and density. Pores are randomly distributed within the solid bone matrix until the desired pore density is reached. Spectroscopy is performed with $0.5 \mathrm{MHz}$ frequency intervals in the $1-8 \mathrm{MHz}$ range with the emitted plane wave as Gaussian ultrasonic pulses with a central frequency within the spectroscopy range and $-6 \mathrm{~dB}$ bandwidth of 20 percent. In order to take the effect of randomness into account, for the same pore density and diameter different realizations are generated and tested for each central frequency.

In order to ignore the effect of reflection, Perfectly Matched Layer (PML) boundary conditions are considered at both ends of the geometry in the direction of wave propagation. The PML thickness is of 15 times that of the wavelength. Symmetry boundary conditions are applied in the direction perpendicular to the wave propagation direction so that plane wave conditions can be assumed and the effect of diffraction can be avoided. The grid step is selected as $10 \mu \mathrm{m}$ in $2 \mathrm{D}$ simulations, resulting in a spatial sampling of over 50 points per wavelength [16].

The attenuation value for the propagated signal within the porous structures is calculated through Time-Distance Matrix Approach (TDMA) described in [26].

\section{Mathematical and Statistical Models}

Physical and biology systems can be represented using mathematical models. These allow one to investigate hypotheses regarding the underlying physical process. A mechanistic model hypothesizes the relationships between physically interpretable parameters and variables, while a phenomenological model captures the qualitative trends of the desired dynamics. Here, we are interested in the process of wave propagation in highly porous structures.

We use a phenomenological model in the form of a power law, developed in [26], to describe frequency dependent attenuation in porous, heterogeneous material mimicking cortical bone. Here model parameters, $a$ and $b$, are presumed to be functions of the micro-architectural parameters, pore diameter $(\phi)$ and pore density $(\rho)$. Thus, our mathematical model for the attenuation coefficient is

$$
\alpha(f)=a(\phi, \rho) f^{b(\phi, \rho)}+c,
$$

where $f$ is frequency, and model parameters are $\boldsymbol{\theta}=\left[\begin{array}{lll}a & b & c\end{array}\right]$. Note here, only $a$ and $b$ are considered to be functionally dependent on diameter and density as the model is most sensitive to these estimates [26].

In order to use model parameter estimates to make meaningful inferences, one must account for the error incurred in the data collection procedure. This is done by specifying a statistical model, which represents the observation process. To account for observational error, we consider the following statistical error model,

$$
Y(f)=\alpha\left(f, \boldsymbol{\theta}_{0}\right)+\mathcal{E}(f),
$$

where $Y(f)$ is a random variable, $\boldsymbol{\theta}_{0}$ is the nominal parameter vector, and the $\mathcal{E}$ are assumed to be independent and identically distributed with mean 0 and variance $\sigma_{0}^{2}$. A realization of this statistical error model is given by

$$
y(f)=\alpha\left(f, \boldsymbol{\theta}_{0}\right)+\epsilon(f), \quad f \in\left[f_{0}, f_{F}\right],
$$

where $\epsilon$ is a specific realization of the random variable $\mathcal{E}$. This absolute error specification is reasonable as the data is numerically simulated, so one expects the errors in simulation to be drawn from the same distribution. Correct specification of both the mathematical and statistical model is neccessary to ensure accuracy of the inferences made regarding parameter estimates.

\section{INVERSE PROBLEM}

We consider an absolute error model in (3). Therefore, following [30], [31] we use an OLS formulation of the inverse problem to attain parameter estimates. Solving this inverse problem corresponds to minimizing the sum of squared errors between the data and model output when data observations are weighted equally.

A realization of the random variable OLS estimator is given by

$$
\hat{\boldsymbol{\theta}}_{O L S}^{N}=\operatorname{argmin}_{\boldsymbol{\theta}} \sum_{j=1}^{N}\left[y_{j}-\alpha\left(f_{j}, \boldsymbol{\theta}\right)\right]^{2},
$$

where the data, $y_{j}$, is realization of the random variable $Y_{j}, \alpha\left(f_{j}, \boldsymbol{\theta}\right)$ is the model output, and $N$ is the number of frequency points.

\section{RESUlts AND DISCUSSION}

In order to infer micro-architectural properties using the phenomenological model two inverse problems are solved. First, one determines model parameter estimates, $\hat{\boldsymbol{\theta}}=\left[\begin{array}{lll}\hat{a} & \hat{b} & \hat{c}\end{array}\right]$, given simulated attenuation data. As mentioned above, this is done using an ordinary least squares (OLS) formulation of the inverse problem. A graph of the resulting parameter estimates versus pore diameter and pore density is given in Figs. 1 and 2 . 


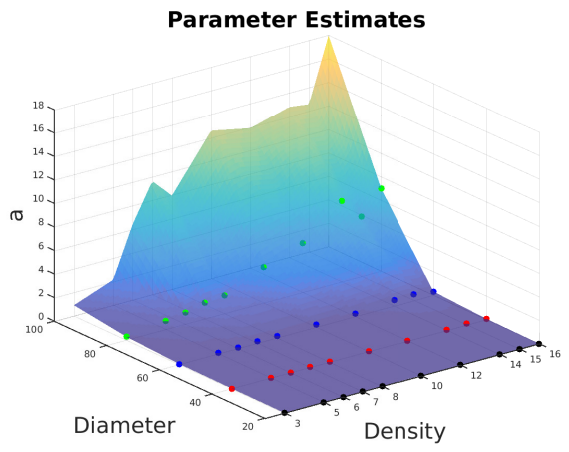

Fig. 1. Parameter $a$ estimates versus pore diameter ([2 $\left.\left.\begin{array}{lllll}20 & 40 & 60 & 80 & 100\end{array}\right] \mu m\right)$ and pore density $\left(\left[\begin{array}{llllllllll}3 & 5 & 6 & 7 & 8 & 10 & 12 & 14 & 15 & 16\end{array}\right] \mathrm{pore} / \mathrm{mm}^{2}\right)$.

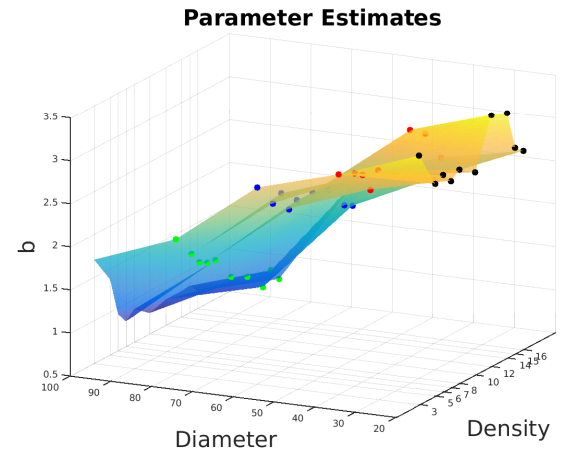

Fig. 2. Parameter $b$ estimates versus pore diameter ([20 406080100$] \mu m)$ and pore density ([3 5567781012141516$\left.] \mathrm{pore} / \mathrm{mm}^{2}\right)$.

Secondly, the relationship between these model parameter estimates, $\hat{a}$ and $\hat{b}$, and the micro-architectural parameters, pore density $(\rho)$ and diameter $(\phi)$ is estimated. To begin, we examine the relationship between the estimates, $\hat{b}$, and pore diameter, $\phi$. Fig. 2 indicates that $\hat{b}$ increases in a linear trend as pore diameter decreases. As a result, we fit a linear model of the average parameter estimate, $\hat{b}_{\text {av }}$ (where we are averaging over densities), as a function of pore diameter. This linear model is given as

$$
\hat{b}_{\mathrm{av}}=-0.0269 \phi+3.8047,
$$

and the resulting fit is depicted in Fig. 3 .

In order to get an idea of how well this linear relationship predicts pore diameter, (5) is used on the parameter estimates from our numerically generated datasets (where the true diameter is known) in order to predict a diameter, $\hat{\phi}$. Note there are ten datasets (for the 10 pore densities) for each pore diameter, which results in ranges of predicted diameters for each true diameter value. These results are given in Tab. I.

Thus, the estimates $\hat{b}$ have been used to infer pore diameter. However, porosity is characterized by both pore density and diameter. In order to address this, we next consider the relationship between the estimates, $\hat{a}$ and pore density, $\rho$. In doing this, we assume accurate knowledge of pore diameter (derived from the linear relationship of $\hat{b}_{\mathrm{av}}$ to $\phi$ ). Again, we see

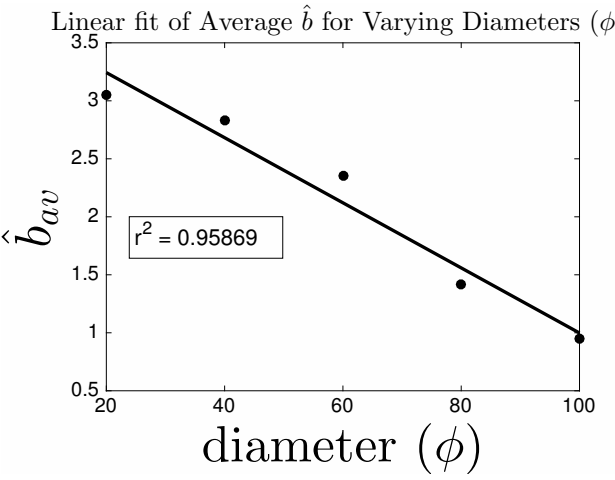

Fig. 3. Linear fit of average $\hat{b}$ values versus pore diameter ([20 406080 100] $\mu m)$.

TABLE I

ESTIMATED PORE DIAMETER, $\hat{\phi}$, USING $\hat{b}_{\mathrm{AV}}$ VERSUS TRUE PORE DIAMETER, $\phi_{0}$

\begin{tabular}{|c|c|}
\hline$\phi_{0}(\mu m)$ & $\hat{\phi}(\mu m)$ \\
\hline 20 & $13-36$ \\
40 & $24-50$ \\
60 & $36-66$ \\
80 & $62-102$ \\
100 & $75-111$ \\
\hline
\end{tabular}

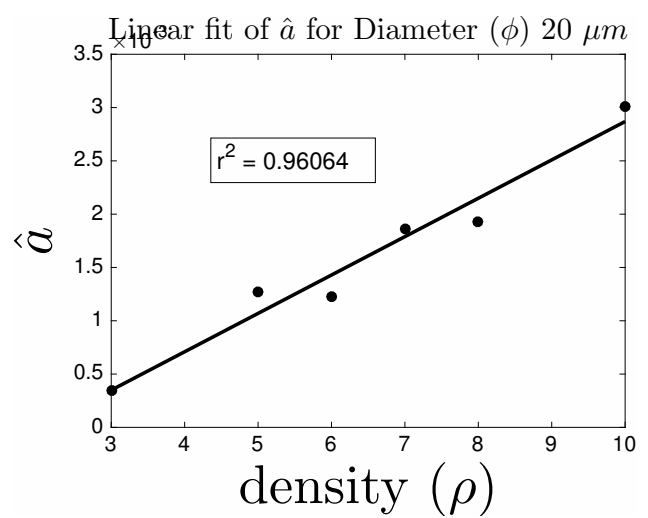

Fig. 4. Linear fit of $\hat{a}$ values for diameter $=20 \mu m$ versus pore density ([3 $\left.56 \begin{array}{lll}6 & 8 & 10\end{array}\right]$ pore $/ \mathrm{mm}^{2}$ ).

that within a given diameter, estimates of $a$ increase linearly as density increases (Fig. 1). The linear fits for pore diameters of $20 \mu \mathrm{m}, 60 \mu \mathrm{m}$, and $100 \mu \mathrm{m}$ are depicted in Figs. 4 - 6, with a similar trend noticed for diameters of 40 and $80 \mu \mathrm{m}$. One should note that in fitting these linear functions, only the main densities of interest, which range from $3-10 \mathrm{~mm}^{2}$, were considered as higher densities did not follow the linear trend. Again, to better understand the precision of pore density prediction, these linear models are used on the numerically generated data (where true density is known). These results are given in Tab. II, where one can compare the true density, $\rho_{0}$, to the estimated density, $\hat{\rho}$ for each diameter considered. 


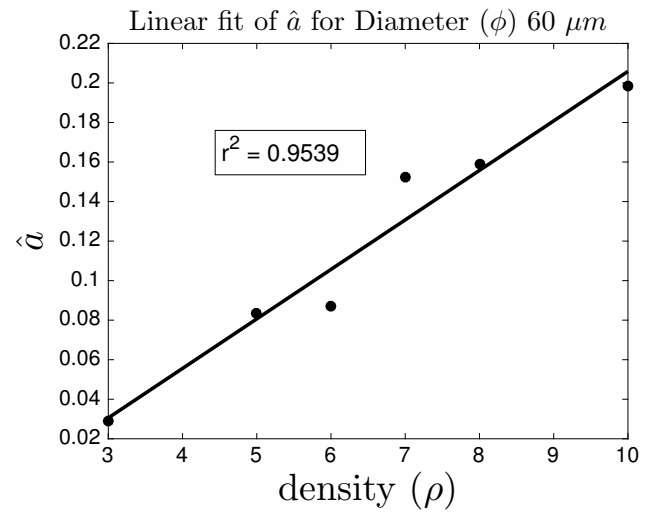

Fig. 5. Linear fit of $\hat{a}$ values for diameter $=60 \mu \mathrm{m}$ versus pore density ([3 5678 10] pore $/ \mathrm{mm}^{2}$ ).

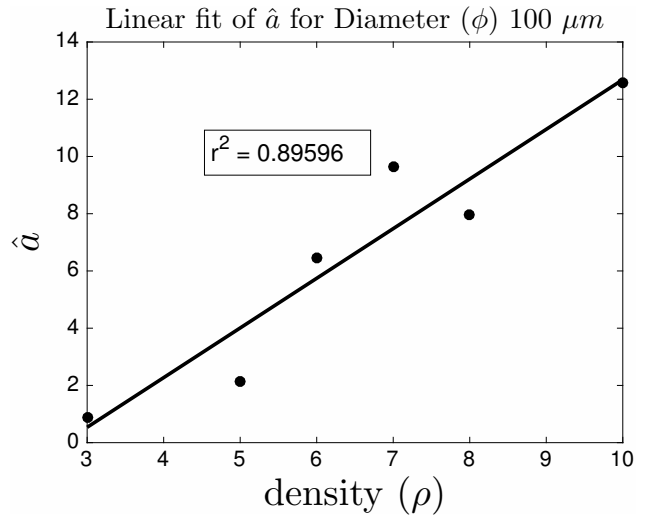

Fig. 6. Linear fit of $\hat{a}$ values for diameter $=100 \mu \mathrm{m}$ versus pore density ([3

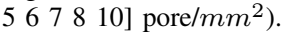

\section{CONCLUSIONS}

The goal of this research was to explore the use of a phenomenological model to infer micro-architectural properties of cortical bone-like structures given frequency dependent attenuation data. We used numerically simulated data from a finite-difference, time domain SimSonic research freeware package, which simulates elastic waves propagating in a heterogeneous media. We determined model parameter estimates by optimizing our model to this data under an OLS formulation of the inverse problem. With these estimates, we then optimized linear functions of our micro-architectural parameters to

TABLE II

ESTIMATED PORE DENSITY, $\hat{\rho}$, USING $\hat{a}$ VERSUS TRUE PORE DENSITY, $\rho_{0}$ FOR PORE DIAMETERS $\phi=\left[\begin{array}{lllll}20 & 40 & 60 & 80 & 100\end{array}\right] \mu \mathrm{m}$

\begin{tabular}{|c|c|c|c|c|c|}
\hline$\rho_{0}$ & \multicolumn{5}{|c|}{$\hat{\rho}$} \\
\hline & $\phi=20$ & $\phi=40$ & $\phi=60$ & $\phi=80$ & $\phi=100$ \\
\hline 3 & 2.9949 & 3.3198 & 2.9329 & 3.7231 & 3.2078 \\
5 & 5.5752 & 4.4730 & 5.1080 & 4.8623 & 3.9335 \\
6 & 5.4306 & 5.8182 & 5.2660 & 5.6542 & 6.4005 \\
7 & 7.2120 & 8.1121 & 7.8744 & 6.6417 & 8.2448 \\
8 & 7.4006 & 6.8936 & 8.1212 & 7.0425 & 7.2708 \\
10 & 10.3866 & 10.3833 & 9.6974 & 11.0762 & 9.9426 \\
\hline
\end{tabular}

these parameter estimates to quantify the relationship between them. Doing so allowed us to infer ranges of porosity given ultrasonic attenuation data.

We determined that model parameter estimates are dependent on the micro-architectural properties of the heterogeneous, porous material. Furthermore, we found that when using numerically generated attenuation data for samples with constant pore size and a given concentration, we could infer ranges of porosity by solving an inverse problem using the phenomenological model. These ranges provide a low resolution way of quantifying porosity in structures mimicking cortical bone. However, the relevance of these ranges for clinical diagnosis remains to be determined. The use of the phenomenological model in this work was based on simulations on 2D structures. Results from 3D simulations in comparison with 2D were used to validate the use of data from 2D simulations. Furthermore, absorption was not considered in the simulations and mathematical model, rather attenuation was considered to be exclusively due to wave scattering. Moreover, all the structures studied were monodisperse random geometries and the relevance of the obtain ranges for pore diameter and density to clinical diagnosis remains to be determined. Future work could incorporate information regarding absorption by altering the numerical simulation for data or by collecting data in vitro.

\section{REFERENCES}

[1] D. M.L. Cooper, C. E. Kawalilak, K. Harrison, B. D. Johnston, and J. D Johnston. Cortical Bone Porosity: What Is It, Why Is It Important, and How Can We Detect It? Current Osteoporosis Reports, 14(5):187-198, 2016.

[2] Roger MD Zebaze, Ali Ghasem-Zadeh, Ann Bohte, Sandra IulianoBurns, Michiko Mirams, Roger Ian Price, Eleanor J. Mackie, and Ego Seeman. Intracortical remodelling and porosity in the distal radius and post-mortem femurs of women: a cross-sectional study. The Lancet, 375(9727):1729-1736, 2010.

[3] Philipp Schneider, Martin Stauber, Romain Voide, Marco Stampanoni, Leah Rae Donahue, and Ralph Müller. Ultrastructural properties in cortical bone vary greatly in two inbred strains of mice as assessed by synchrotron light based micro- and nano-CT. Journal of Bone and Mineral Research, 22(10):1557-1570, 2007.

[4] A Mgeough and B Barker. Age-Related. 75(8), 1993.

[5] Paolo E Palacio-Mancheno, Adriana I Larriera, Stephen B Doty, Luis Cardoso, and Susannah P Fritton. 3D assessment of cortical bone porosity and tissue mineral density using high-resolution $\$ \mu \$ \mathrm{cT}$ : Effects of resolution and threshold method. Journal of Bone and Mineral Research, 29(1):142-150, 2014.

[6] Chamith S Rajapakse, Mahdieh Bashoor-Zadeh, Cheng Li, Wenli Sun, Alexander C Wright, and Felix W Wehrli. Volumetric Cortical Bone Porosity Assessment with MR Imaging: Validation and Clinical Feasibility. Radiology, 276(2):526-35, 2015.

[7] Claudio Ripamonti, Lucia Lisi, Angela Buffa, Saverio Gnudi, and Renata Caudarella. The Trabecular Bone Score Predicts Spine Fragility Fractures in Postmenopausal Caucasian Women Without Osteoporosis Independently of Bone Mineral Density. Medical Archives, 72(1):46, 2018.

[8] J. A. Kanis and C C Glüer. An update on the diagnosis and assessment of osteoporosis with densitometry. Committee of Scientific Advisors, International Osteoporosis Foundation. Osteoporosis international : a journal established as result of cooperation between the European Foundation for Osteoporosis and the National Osteoporosis Foundation of the USA, 11(3):192-202, 2000.

[9] D. Marshall, O. Johnell, and H. Wedel. Meta-analysis of how well measures of bone mineral density predict occurrence of osteoporotic fractures. Bmj, 312(7041):1254-1259, 1996. 
[10] Yohann Bala, Roger Zebaze, Ali Ghasem-Zadeh, Elizabeth J Atkinson, Sandra Iuliano, James M Peterson, Shreyasee Amin, shild Bjørnerem, L Joseph Melton, Helena Johansson, John A Kanis, Sundeep Khosla, and Ego Seeman. Cortical porosity identifies women with osteopenia at increased risk for forearm fractures. Journal of bone and mineral research : the official journal of the American Society for Bone and Mineral Research, 29(6):1356-62, 62014.

[11] Huayue Chen, Xiangrong Zhou, Hiroshi Fujita, Minoru Onozuka, and Kin-Ya Kubo. Age-Related Changes in Trabecular and Cortical Bone Microstructure. International Journal of Endocrinology, 2013:1-9, 2013.

[12] Janardhan Yerramshetty and Ozan Akkus. Changes in Cortical Bone Mineral and Microstructure with Aging and Osteoporosis. pages 105131. 2012.

[13] Georg Osterhoff, Elise F Morgan, Sandra J Shefelbine, Lamya Karim, Laoise M Mcnamara, and Peter Augat. Bone mechanical properties and changes with osteoporosis. Injury, Int. J. Care Injured, 47:S11-S20, 2016.

[14] P. Rüiegsegger, B. Koller, and R. Müller. A microtomographic system for the nondestructive evaluation of bone architecture. Calcified Tissue International, 58(1):24-29, 11996.

[15] Pascal Laugier and Guillaume Haïat, editors. Bone Quantitative Ultrasound. Springer Netherlands, Dordrecht, 2011.

[16] Petro Moilanen, Patrick H.F. Nicholson, Vantte Kilappa, Sulin Cheng, and Jussi Timonen. Assessment of the cortical bone thickness using ultrasonic guided waves: Modelling and in vitro study. Ultrasound in Medicine \& Biology, 33(2):254-262, 22007.

[17] Josquin Foiret, Jean-Gabriel Minonzio, Christine Chappard, Maryline Talmant, and Pascal Laugier. Combined estimation of thickness and velocities using ultrasound guided waves: a pioneering study on in vitro cortical bone samples. IEEE Transactions on Ultrasonics, Ferroelectrics and Frequency Control, 61(9):1478-1488, 2014.

[18] Emmanuel Bossy, Maryline Talmant, and Pascal Laugier. Threedimensional simulations of ultrasonic axial transmission velocity measurement on cortical bone models. 115:2314-2314, 2004.

[19] Luiz Garcia Mandarano-Filho, Mrcio Takey Bezuti, Nilton Mazzer, and Cludio Henrique Barbieri. Influence of cortical bone thickness on the ultrasound velocity. Acta ortopedica brasileira, 20(3):184-90, 2012.

[20] E. C. Rose, M. Hagenmüller, I. E. Jonas, and B. A. Rahn. Validation of speed of sound for the assessment of cortical bone maturity. European Journal of Orthodontics, 27(2):190-195, 12005.

[21] H Sievänen, S Cheng, S Ollikainen, and K Uusi-Rasi. Ultrasound velocity and cortical bone characteristics in vivo. Osteoporosis international : a journal established as result of cooperation between the European Foundation for Osteoporosis and the National Osteoporosis Foundation of the USA, 12(5):399-405, 2001.

[22] Omid Yousefian, Rebekah White, H. Thomas Banks, and Marie Muller. Ultrasonic attenuation spectroscopy and dispersion characteristics in cortical bone. In 2017 IEEE International Ultrasonics Symposium (IUS), pages 1-4. IEEE, 92017.

[23] Emmanuel Bossy, Frdric Padilla, Franoise Peyrin, and Pascal Laugier. Three-dimensional simulation of ultrasound propagation through trabecular bone structures measured by synchrotron microtomography. Physics in Medicine and Biology, 50(23):5545-5556, 122005.

[24] Didier Cassereau, Pierre Nauleau, Quentin Grimal, Jean-Gabriel Minonzio, Aniss Bendjoudi, Emmanuel Bossy, and Pascal Laugier. Coupling of finite difference elastodynamic and semi-analytic Rayleigh integral codes for the modeling of ultrasound propagation at the hip. The Journal of the Acoustical Society of America, 133(5):3498-3498, 5 2013.

[25] F. Padilla, E. Bossy, G. Haiat, F. Jenson, and P. Laugier. Numerical simulation of wave propagation in cancellous bone. Ultrasonics, 44:e239-e243, 122006.

[26] Omid Yousefian, R. D. White, Yasamin Karbalaeisadegh, H. T. Banks, and Marie Muller. The effect of pore size and density on ultrasonic attenuation in porous structures with mono-disperse random pore distribution: A two-dimensional in-silico study. The Journal of the Acoustical Society of America, 144(2):709-719, 82018.

[27] C David L Thomas, Sophie A Feik, and John G Clement. Increase in pore area, and not pore density, is the main determinant in the development of porosity in human cortical bone. Journal of anatomy, 209(2):219-30, 82006.

[28] Chibuzor T. M. Eneh, Isaac O. Afara, Markus K. H. Malo, Jukka S. Jurvelin, and Juha Töyräs. Porosity predicted from ultrasound backscat- ter using multivariate analysis can improve accuracy of cortical bone thickness assessment. The Journal of the Acoustical Society of America, 141(1):575-585, 12017.

[29] Valrie Bousson, Alain Meunier, Catherine Bergot, ric Vicaut, Maria Augusta Rocha, Maria Helena Morais, Anne-Marie Laval-Jeantet, and Jean-Denis Laredo. Distribution of Intracortical Porosity in Human Midfemoral Cortex by Age and Gender. Journal of Bone and Mineral Research, 16(7):1308-1317, 72001.

[30] H. Thomas Banks, Shuhua. Hu, and W. Clayton Thompson. Modeling and inverse problems in the presence of uncertainty. CRC Press, Taylor and Francis, 2014.

[31] H. T. (Harvey Thomas) Banks and H. T. Tran. Mathematical and experimental modeling of physical and biological processes. CRC Press, 2009. 